\title{
O OBLABI (Laboratório e Observatório de Práticas Inovadoras em Educação) e a pesquisa sobre tecnologias móveis
}

\author{
Luciane Mulazani dos $\operatorname{Santos}^{12}$, Gustavo Leandro da Silveira ${ }^{1}$, Nathiele Costa ${ }^{1}$, \\ Carolina Soares Bueno ${ }^{2}$, Rafael Sato ${ }^{1}$, Elisa Henning ${ }^{1}$ \\ ${ }^{1}$ Departamento de Matemática - Centro do Ciências Tecnológicas - Universidade do \\ Estado de Santa Catarina (UDESC) \\ Rua Paulo Malschitzki, s/n - 89.219-710 - Joinville - SC - Brasil \\ ${ }^{2}$ Programa de Pós-graduação em Ciências e em Matemática - Universidade Federal do \\ Paraná (UFPR) \\ Centro Politécnico - 81.531-980 - Curitiba - PR - Brasil. \\ luciane.mulazani@udesc.br, gustavoldasilveira@gmail.com, \\ nathielecosta@live.com, carolsoaresbueno@gmail.com, \\ rafaeltsato@gmail.com, elisa.henning@udesc.br
}

\begin{abstract}
This paper presents a work in progress that is being developed at Universidade do Estado de Santa Catarina - UDESC as a research project in the area of Mathematics Education. These are studies in a Observatory and Laboratory of Innovative Practices in Education (OBLABI) on selection, experimentation and development of learning objects of Mathematics and Statistics to be used on mobile devices such as tablets and smartphones, at all levels of education, from primary to higher education. In this article, we present a discussion about the potential and challenges of using mobile technologies in education.
\end{abstract}

Resumo. Este artigo apresenta um trabalho em andamento que vem sendo desenvolvido na Universidade do Estado de Santa Catarina - UDESC como um projeto de pesquisa na área de Educação Matemática. Tratam-se de estudos realizados pelo grupo do OBLABI (Observatório e Laboratório de Práticas Inovadoras em Educação) sobre seleção, experimentação e desenvolvimento de objetos de aprendizagem de conteúdos matemáticos e estatísticos a serem utilizados em dispositivos móveis, tais como tablets e smartphones, nos níveis de ensino Fundamental, Médio e Superior. Neste artigo, apresentamos uma discussão a respeito das potencialidades e desafios da utilização de tecnologias móveis na Educação.

\section{Inovação em educação: indicações para o futuro}

Professores de disciplinas de conteúdos de Matemática de todos os níveis de ensino costumam enfrentar desafios nos processos de ensino e aprendizagem relacionados com as dificuldades de se ensinar e de se aprender Matemática. É comum, por exemplo, encontrarmos alunos que dizem que não gostam da Matemática, que a Matemática é para poucos, que a Matemática serve para coisa alguma e que é difícil de aprender. 
Pesquisadores e professores de disciplinas de conteúdos matemáticos preocupam-se, em suas reflexões e práticas didáticas, com a mudança desse quadro visando ao sucesso na aprendizagem. Estudam-se, assim, métodos de avaliação, desenvolvimento de novas práticas, criação de novos recursos didáticos, utilização da tecnologia e mais uma infinidade de recursos que podem ajudar o professor na tarefa de ensinar Matemática e o aluno na missão de aprender Matemática. Percebe-se, nesse contexto e no âmbito da Educação Matemática, uma crescente preocupação com a inserção da tecnologia no ambiente escolar na forma de utilização de objetos de aprendizagem inovadores.

Objetos de aprendizagem são importantes nos processos de ensino e aprendizagem que utilizam o computador em sua mediação (GIBBONS, 2000) e que se apoiam nas Tecnologias de Informação e Comunicação, sendo recursos digitais reutilizáveis (WILEY, 2002) em diferentes situações de aprendizagem (BECK, 2002) que contêm as informações para as quais significados são produzidos no processo de construção de conhecimento (Santos, 2007) e podem ser utilizados tanto no ensino a distância quanto no ensino presencial.

Em tempos de frequente e popular utilização de internet móvel em smartphones e tablets para comunicação em redes sociais, não se pode deixar de lado o potencial dos dispositivos móveis em atividades de ensino e aprendizagem tanto dentro da escola como fora dela.

Jogando luz nesse assunto, a recente divulgação do Horizon Report 2013 voltado ao ensino superior feita pelo NMC (New Media Consortium) e pela EDUCASE Learning Initiave, organizações norte-americanas dedicadas ao estudo das tendências na educação, aponta seis desafios, seis tendências e seis tecnologias emergentes que deverão se tornar populares até 2018. Neste que é um documento anual elaborado por especialistas em educação, tecnologia e futuro e por escritores e pensadores, são apresentados importantes indicativos para caminhos de pesquisa que as universidades devem trilhar ao longo dos próximos cinco anos. Dentre eles, destacamos os apresentados no quadro abaixo:

Quadro 1 - Desafios, tendências e tecnologias emergentes que deverão se tornar populares até 2018, segundo Horizon Report, 2013 para as universidades.

\begin{tabular}{l|l|l}
\hline $\begin{array}{l}\text { Tecnologias com maior potencial de aplicação em processos de ensino e } \\
\text { aprendizagem }\end{array}$ & \multicolumn{1}{c}{ Para quando? } & \multicolumn{1}{c}{ Como } \\
\hline O quê? & em 1 ano & $\begin{array}{l}\text { Desenvolvimento de } \\
\text { aplicativos educacionais para } \\
\text { serem usados em dispositivos } \\
\text { móveis }\end{array}$ \\
\hline Aplicativos móveis & em 1 ano & $\begin{array}{l}\text { Utilização de equipamentos } \\
\text { portáteis, versáteis e cada vez } \\
\text { mais baratos para } \\
\text { aprendizagem em sala de aula } \\
\text { de maneira compartilhada. }\end{array}$ \\
\hline Learning analytics & de 2 a 3 anos & $\begin{array}{l}\text { Para acompanhar os rastros de } \\
\text { aprendizagem deixados pelos }\end{array}$ \\
\hline
\end{tabular}


alunos em ambientes online.

Tendências para a Educação relacionadas com as tecnologias indicadas

Novas fontes de informação online que poderão ser utilizadas para personalizarem e avaliarem os processos de ensino e aprendizagem. Registro dos rastros digitais de aprendizagem

Novo papel para o professor frente à transformação tecnológica dos recursos didáticos e dos ambientes de aprendizagem

Novo paradigma educacional apoiado na vivência online e colaborativa

Desafios indicados às Universidades balisados pelas tecnologias emergentes e pelas tendências prospectadas à Educação

Capacitação dos professores para a era digital

Quebra de resistência quanto ao uso de dispositivos e aplicativos tecnológicos nos processos de ensino e aprendizagem

Personalização de recursos didáticos e de prática didática considerando $\mathrm{o}$ público de alunos adaptados à era digital

A Organização das Nações Unidas para a Educação, a Ciência e a Cultura UNESCO (United Nations Educational, Scientific and Cultural Organization) divulgou, no início de 2013, um relatório a respeito de políticas para a aprendizagem móvel. Neste relatório, apresenta diretrizes e políticas para que os dispositivos móveis (como tablets e smartphones) sejam utilizados como recursos tecnológicos que apoiem os processos de ensino e aprendizagem no contexto da busca por uma educação de qualidade no mundo todo. No documento, são apresentadas 10 recomendações para os governos implantarem políticas públicas que utilizem dispositivos móveis como recurso nas salas de aula e 13 motivos para que estes recursos sejam aceitos como aliados na busca por uma educação de qualidade. Reproduzimos esses importantes pontos no quadro abaixo:

Quadro 2 - Políticas para a aprendizagem móvel segundo relatório da UNESCO, de 2013.

10 recomendações para os governos implantarem políticas públicas que utilizem dispositivos móveis como recurso nas salas de aula

Criar ou atualizar políticas ligadas ao aprendizado móvel

Conscientizar sobre sua importância

Expandir e melhorar opções de conexão

Ter acesso igualitário

Garantir equidade de gênero

Criar e otimizar conteúdo educacional

Treinar professores

Capacitar educadores usando tecnologias móveis

Promover o uso seguro, saudável e responsável de tecnologias móveis

Usar tecnologia para melhorar a comunicação e a gestão educacional

13 motivos para usar os dispositivos móveis como recursos pedagógicos

Amplia o alcance e a equidade em educação

Melhora a educação em áreas de conflito ou que sofreram desastres naturais

Assiste alunos com deficiência

Otimiza o tempo na sala de aula 


\begin{tabular}{l}
\hline Permite que se aprenda em qualquer hora e lugar \\
\hline Constroi novas comunidades de aprendizado \\
\hline Dá suporte a aprendizagem in loco \\
\hline Aproxima o aprendizado formal do informal \\
\hline Provê avaliação e feedback imediatos \\
\hline Facilita o aprendizado personalizado \\
\hline Melhora a aprendizagem contínua \\
\hline Melhora a comunicação \\
\hline Maximiza a relação custo-benefício da educação \\
\hline
\end{tabular}

Observando as indicações do Horizon Report e do relatório da UNESCO para aprendizagem móvel, percebe-se o foco dado à necessidade de existência de políticas educacionais que incentivem o uso das tecnologias móveis em sala de aula. Isso ressalta a importância de que sejam revistas as políticas já existentes em busca de atualização considerando que estas foram determinadas em um tempo onde as tecnologias móveis não eram tão acessíveis como hoje. Além disso, há um importante foco, nestes documentos, na necessidade de formação dos professores para o uso destas tecnologias: tanto a formação inicial como a continuada devem contemplar o uso que fazemos das Tecnologias de Informação e Comunicação em estreita relação com a utilização dos dispositivos móveis nas escolas.

Ao lado da experiência com o trabalho com a formação inicial de professores de Matemática no curso de Licenciatura em Matemática da Universidade do Estado de Santa Catarina (UDESC), as considerações apresentadas no Horizon Report 2013 e nas Diretrizes de políticas para a aprendizagem móvel, publicada pela UNESCO em 2013, contribuíriam para a concepção e criação do OBLABI - Observatório e Laboratório de Práticas Inovadoras em Educação, bem como para definição de uma de suas ações: estudar, selecionar, experimentar e desenvolver objetos de aprendizagem de Matemática e Estatística que podem ser utilizados em dispositivos móveis, tais como tablets e smartphones, nos níveis de ensino Fundamental, Médio e Superior. Entendemos que, com o trabalho de pesquisa e desenvolvimento de práticas realizado pelo grupo de pesquisadores do OBLABI - que envolve professores e alunos do curso - a universidade cumpre um importante papel na formação de professores para o uso da tecnologia móvel na Educação.

Nesse workshop, pretendemos apresentar uma discussão a respeito das indicações para o futuro para a Educação, a partir de considerações sobre os documentos Horizon Report 2013 e Diretrizes da UNESCO de políticas para a aprendizagem móvel e também a respeito das atividades de pesquisa e práticas pedagógicas que estão sendo realizadas no OBLABI.

\section{Caminhos para as tecnologias móveis na Educação}

Pesquisadores, como Moran, já estão atentos às mudanças nos processos de ensino e aprendizagem por conta da inserção das novas tecnologias baseadas na utilização de dispositivos móveis.

No Brasil, os smartphones e os tablets ainda estão numa fase de experimentação dentro das escolas. Trazem desafios complexos. 
São cada vez mais fáceis de usar, permitem a colaboração entre pessoas próximas e distantes, ampliam a noção de espaço escolar, integrando os alunos e professores de países, línguas e culturas diferentes. E todos, além da aprendizagem formal, têm a oportunidade de se engajar, aprender e desenvolver relações duradouras para suas vidas. Ensinar e aprender podem ser feitos de forma muito mais flexível, ativa e focada no ritmo de cada um. (MORAN, 2013)

Ainda de acordo com o autor,

As tecnologias móveis trazem enormes desafios, porque descentralizam os processos de gestão do conhecimento: podemos aprender em qualquer lugar, a qualquer hora e de muitas formas diferentes. Podemos aprender sozinhos e em grupo, estando juntos fisicamente ou conectados. (MORAN, 2013)

No Brasil, os tablets já estão chegando às escolas públicas via Projeto Educação Digital - política para computadores interativos e tablets - implementado pelo MEC Ministério da Educação. Essa ação faz parte do projeto de oportunizar aos professores a utilização de recursos didáticos inovadores nos processos de ensino e aprendizagem bem como capacitá-los para o uso das TICs - Tecnologias de Informação e Comunicação de forma que tais dispositivos móveis apoiem o desenvolvimento de atividades didáticas dinâmicas e criativas de maneira autônoma.

Segundo dados do Ministério da Educação (MEC, 2012), foi previsto - e já está sendo destinado - um orçamento de cerca de R $\$ 150$ milhões para a compra de 600 mil tablets que serão enviados aos professores que atuam nas escolas públicas do Brasil. É uma importante ação para o fortalecimento das políticas públicas de educação e para inserção desses professores nas novas tendências e desafios propostos para a Educação para os próximos anos no que se refere à era digital.

É muito importante que a gente construa uma estratégia sólida para que a escola possa formar, preparar essa nova geração para o uso de tecnologias da informação. (...) Estamos definindo que, na educação, a inclusão digital começa pelo professor. (Entrevista com o Ministro da Educação Aloizio Mercadante, 2012)

"As inovações mais promissoras encontram-se em escolas que têm tecnologias móveis na sala de aula utilizadas por professores e alunos" (MORAN, 2013). Estando em sala de aula os dispositivos móveis para serem utilizados na educação, é preciso que repensemos a educação para que o professor atue como mediador organizando processos de ensino e aprendizagem mais abertos e colaborativos.

A proposta de pesquisa do OBLABI é juntar-se às pesquisas já existentes para compor um cenário que discuta e, mais do que isso, proponha soluções para a utilização 
dos dispositivos móveis nas salas de aula utilizando seus potenciais máximos visando ao ensino e à aprendizagem de conteúdos de Matemática dos níveis de ensino Fundamental, Médio e Superior.

Há interessantes e eficientes aplicativos desenvolvidos para ensinar e aprender utilizando dispositivos móveis dos mais diferentes sistemas operacionais. Pode-se, por exemplo, aprender uma nova língua, fazer cursos inteiros, tirar dúvidas utilizando aplicativos que rodam nos smartphones e nos tablets. Algumas instituições de ensino disponibilizam seus cursos também para estas plataformas. Exemplos podem ser consultados na compilação feita por The New Media Consortium (2013, pp. 17-19) apresentada no Horizon Report 2013. Estão sendo estudadas soluções semelhantes a essas, no âmbito da Educação Matemática, pelo Observatório e Laboratório de Práticas Inovadoras em Educação.

Moran conclui, em sua discussão acerca do uso de aplicativos e dispositivos móveis em processos de ensino e aprendizagem, que o sucesso das ações educativas dependem de como os professores integram as novas tecnologias aos seus objetivos e propostas de ensino reforçando que "ainda precisamos experimentar muito para encontrar caminhos de integração que nos permitam avanços significativos na escola e na vida". Esse projeto de pesquisa propõe-se a contribuir nessa importante questão, prospectando soluções tecnológicas, propondo ações didáticas e de formação de professores, além de desenvolver objetos de aprendizagem para dispositivos móveis.

O sucesso será alcançado não somente com o desenvolvimento de novos aplicativos, mas também com a organização das soluções já existentes acompanhadas de recomendações e análises acerca de seus potenciais de utilização. Para bem usar os recursos, é preciso saber que eles existem e onde encontrá-los; bem-vindo é também o compartilhamento de experiências de uso como forma de construir novas práticas docentes, função a que se propõe o OBLABI no sentido exposto por Lorenzato (2006) de ser "um espaço para facilitar, tanto ao aluno como ao professor, questionar, conjecturar, procurar, experimentar, analisar e concluir, enfim, aprender e principalmente aprender a aprender".

\section{Considerações finais}

No OBLABI, investigamos a utilização de objetos de aprendizagem em dispositivos móveis (de diferentes arquiteturas e com diferentes sistemas operacionais) com o objetivo de desenvolvermos novos objetos de aprendizagem e aplicativos para o ensino e aprendizagem de conteúdos de Matemática e Estatística. Fazem parte da equipe, dois professores com formação em Educação Matemática, uma professora com formação em Estatística, uma professora com formação em Estatística, dois alunos de iniciação científica do curso de Licenciatura em Matemática da UDESC e uma aluna de mestrado de Educação em Ciências e em Matemática da UFPR (Universidade Federal do Paraná). Contamos com apoio técnico de profissionais da área de tecnologia e apoio financeiro da FAPESC - Fundação de Amparo à Pesquisa e Inovação do Estado de Santa Catarina e da FITEJ - Fundação Instituto Tecnológico de Joinville. As pesquisas estão em andamento - iniciaram no final de 2013 - e estão sendo realizados estudos sobre as potencialidades e desafios das tecnologias móveis para a educação e estão sendo 
estudados alguns aplicativos para ensino de matemática e estatística. Pretendemos ter como produto de pesquisa, além de um arcabouço teórico sistematizado sobre a utilização da tecnologia móvel em sala de aula, a apresentação de novos objetos de aprendizagem e a catalogação de recursos já disponíveis. É objetivo do OBLABI atuar nas seguintes contribuições científicas ou tecnológicas: desenvolvimento de um produto tecnológico; desenvolvimento de novas práticas pedagógicas; inovação tecnológica na educação; consolidação do uso dos tablets e smartphones nas escolas como recurso educacional e ampliação da pesquisa na área de tecnologia móvel para a educação.

O trabalho de pesquisa e de desenvolvimento de práticas do OBLABI está balisado nas indicações sobre o uso das Tecnologias de Informação e Comunicação para uma educação de qualidade, sobre uso responsável e pedagógico dos dispositivos móveis na educação e sobre a necessidade de formação inicial e continuada de qualidade para os professores que atual em todos os níveis de ensino. Os trabalhos do OBLABI estão em seus passos iniciais, mas já começamos a desenvolver os primeiros recursos em busca da concretização do projeto: nesta primeira fase, estão sendo avaliados aplicativos e objetos de aprendizagem para o ensino de estatística nos anos iniciais do ensino fundamental, com foco na alfabetização matemática; para o ensino de estatística no ensino superior; para o ensino de geometria na educação básica e no ensino superior; para alfabetização matemática. Além disso, está em desenvolvimento o site do OBLABI que servirá como repositório de objetos de aprendizagem que podem ser utilizados em dispositivos móveis e de sequências didáticas que indicarão como os professores e alunos podem trabalhar com esses recursos com informações também a respeito das diferentes plataformas, sistemas operacionais e arquiteturas de dispositivos móveis que podem ser utilizados em cada caso. A previsão é que o site do OBLABI, nestas condições, tenha seu acesso liberado para consulta geral a partir de fevereiro de 2015.

\section{Referências}

Beck, R. J. (2002) "Learning Objects: What?" Center for Internation Education, University of Winsconsin, Milwaukee.

Gibbons, A. S.; Nelson J.; Richards, R. "The nature and origin of instructional objects". http://reusability.org/read/chapters/gibbons.doc, Maio 2014.

Lorenzato, S. (2006) "O Laboratório de Ensino de Matemática na formação de professores". 1. ed. Autores Associados.

Moran, J. M. "Tablets e netbooks na educação". http://www.eca.usp.br/moran/tablets.pdf, Maio 2014.

Moran, J. M.; Masetto, M.; Behrens, M. "Novas tecnologias e mediação pedagógica". 12. ed. São Paulo: Papirus.

SANTOS, L. M. Produção de Significados para Objetos de Aprendizagem: de autores e leitores para a Educação Matemática. Dissertação (Mestrado em Educação). Curitiba: UFPR, 2007

The New Media Consortium. (2013) "Horizon Report 2013". http://www.nmc.org/pdf/2013-horizon-report-HE.pdf. Maio 2014. 
UNESCO. (2013) "Diretrizes de Políticas para Aprendizagem Móvel" http://www.bibl.ita.br/UNESCO-Diretrizes.pdf. Maio 2014.

Wiley, D. A. Connecting learning objects to instructional design theory: a definition, a metaphor, and a taxonomy. http://reusability.org/read/chapters/wiley.doc. Maio 2014. 